Debate

Series editors: Susan Lightman \& Peter McCluskey

The use of radiotherapy in managing patients with thyroid orbitopathy is controversial. There are widely divergent results from studies of its effectiveness in treating patients and its place in management of thyroid orbitopathy remains uncertain. In this clinical controversy two groups involved in studies ex ploring the effectiveness of radiotherapy in thyroid orbitopathy discuss their views of the role of radiotherapy in managing patients with thyroid orbitopathy.

Correspondence to: Dr Alan A McNab

Kimberly P Cockerham, John S Kennerdell, Allegheny General Hospital, 420 East North Avenue, Pittsburgh, PA 15212-9986, USA

Accepted for publication 6 August 2001

CONTROVERSIES IN OPHTHALMOLOGY

\title{
Does radiotherapy have a role in the management of thyroid orbitopathy? View 1
}

\author{
Kimberley P Cockerham, John S Kennerdell
}

$\mathrm{T}$

hroid orbitopathy (thyroid associated orbitopathy or Graves' ophthalmopathy) is a common cause of proptosis, eyelid retraction, orbital congestion, and motility disturbances in adults. Each patient experiences a unique combination of symptoms and signs for an unpredictable duration and with varying severity. Although orbital manifestations typically improve within 2-5 years, these years feel like a century to the extremely uncomfortable and discontented patients. In addition, visual loss due to optic nerve compression does occur. Radiotherapy has a key role in the management of moderate to severe inflammatory symptoms and is effective treatment for optic nerve compromise.

Our understanding of the link between systemic thyroid disease and orbitopathy has expanded greatly over the past 20 years, although the precise pathogenesis remains uncertain. An unknown mechanism allows thyroid antigen to stimulate the immune system and produce antibodies to the thyroid stimulating hormone (TSH) receptor and other antigens that alter the release of thyroid hormones. Shared orbit thyroid antigens result in the production of activated $\mathrm{T}$ lymphocytes that invade the orbital connective tissue. ${ }^{12}$ Orbital fibroblasts proliferate, resulting in increased synthesis and release of glycosaminoglycans. Locally produced cytokines amplify the inflammatory response. This combined cell mediated and humoral response results in inflammatory cell migration and production of oedema in the orbit. The result is thickening of extraocular muscle and an increase in orbital fat volume. External beam radiation can intervene in this process by arresting the fibroblast proliferation and diminishing the orbital inflammation permanently.

\section{Early signs and symptoms}

Symptoms and subtle signs of thyroid orbitopathy (TO) are often present for many years before diagnosis. Common and non-specific symptoms include tearing, irritation, aching, and photophobia. Early signs include conjunctival injection, periorbital puffiness, abnormal tear break up time, superficial punctate keratitis, and elevation of intraocular pressure. Eyelid retraction is a common early feature of hyperthyroidism, but can occur in euthyroid or hypothyroid patients with orbitopathy. The classic stare of bilateral, symmetric upper, and lower lid retraction may abate after thyroid function stabilises.

After identification of early symptoms and signs of TO, observation and patient education are indicated. Reassurance and description of the natural course of the disease are helpful. Lubricating drops, cool compresses, and sunglasses will improve symptoms during the day. Elevating the head of the bed and taping the eyelids will minimise periorbital oedema and irritation due to nocturnal lagophthalmos. Stable, significant eyelid retraction can be improved with appropriate surgical therapy. Corticosteroids and external beam radiation are never indicated for these early mild TO symptoms and signs.

\section{Dysmotility}

Transient diplopia is very common and can progress to constant diplopia. Difficulties with fusion are usually worse in the morning because of fluid accumulation in the muscles that occurs with recumbency. Infiltration with inflammatory cells and fluid is typically followed by fibrosis which may create a permanent motility restriction. The inferior rectus and medial rectus are most commonly involved resulting in noncomitant esotropia or hypotropia, respectively. Forced duction testing is usually positive as a result of fibrotic contraction. Computed tomography (CT) imaging reveals bilateral, typically asymmetric, enlargement of extraocular muscles. Radiation can improve motility during the active inflammatory phase, but is ineffective against fibrotic extraocular muscle restriction. ${ }^{4-8}$

Most chronic motility disturbances caused by fibrosis are not radiosensitive. Radiation may improve motility in actively inflamed patients but rarely resolves the double vision entirely. Restrictive dysmotility is one of the reasons for the high rate of surgery $(25-90 \%)$ previously noted following radiotherapy. ${ }^{4-68}$ In the recent study by Mourits, et al improvement in motility was the only difference between the group receiving radiation and the sham irradiation group. Twenty five per cent of patients receiving radiation were spared strabismus surgery, but $11 / 17$ had an improvement in the field of binocular vision. ${ }^{8}$

For the vast majority of patients with dysmotility, the patient should be observed for at least 6 months and then offered strabismus surgery when inflammation has subsided and the restrictive component has stabilised.

\section{Proptosis}

Proptosis, the classic feature of thyroid orbitopathy, is actually less prevalent than eyelid retraction. It is caused by infiltration of the eye muscles with inflammatory cells and/or an increase in fat volume, resulting in forward displacement of the globe. Proptosis is usually axial and associated with increased resistance to retropulsion of the globe. Proptosis does not respond to external beam radiation; improvements of only $1-2 \mathrm{~mm}$ are typical in our experience. In several published series proptosis improved in $23-51 \%$ of patients ${ }^{4-6}$; in other studies, no significant change in proptosis occurred. ${ }^{78}$ 
Table 1 Benefits of external beam radiation for thyroid orbitopathy

Directly treats the inflammation of thyroid orbitopathy

Can be utilised in the active phase of disease when surgery is not a good option

Minimises congestion, ache, injection and periorbital oedema

Decreases proptosis and dysmotility caused by acute inflammation

Alleviates visual dysfunction caused by acute optic nerve compression

The risks of general anaesthesia are eliminated

No bruising, swelling, or discomfort

Hospitalisation not required

Safe with minimal side effects in non-diabetic adults

We have used external beam radiation to quieten inflammation so that orbital decompression can be performed in a more timely fashion. No studies have addressed the effectiveness of this combination technique to minimise the duration of the active phase in patients with moderate to severe inflammation. Radiation has no role in the treatment of chronic proptosis.

\section{Orbital inflammation and congestion}

Some patients with TO experience more significant inflammatory symptoms and signs. Rather than mild conjunctival injection over the recti muscle insertions, interpalpebral chemosis and severe periorbital oedema with erythema develop. Increased orbital volume secondary to inflammation is believed to impede venous outflow, which further aggravates congestion and the resulting proptosis. So, although inflammation and congestion are two distinct processes, they are intimately related.

Acute, severe orbital inflammation is effectively treated with corticosteroids combined with radiotherapy. ${ }^{4-610}{ }^{11}$ We do not routinely use pulse intravenous steroids but they have been effectively used by others. ${ }^{12}{ }^{13}$ We prescribe a starting dose of $80 \mathrm{mg}$ of prednisone. The high dose is maintained for at least 1 week and then tapered slowly over 3 months. Care must be taken to tailor dosing to the individual's clinical response. Many patients cannot tolerate the side effects of high dose prednisone and/or are unable to be tapered off the medication without recurrence of symptoms and signs. Low dose radiation (20 Gy) is indicated in these patients to counteract inflammation. Prednisone should be continued during the radiation therapy. This combination of corticosteroids and radiation is only effective during the active phase of inflammation so appropriate patient selection is essential. ${ }^{14-16}$ Patients also should be counselled that further surgery might be necessary if restrictive myopathy or significant proptosis is present (Table 1 ).

A recent study by Mourits and colleagues disputes the effectiveness of external beam irradiation for soft tissue inflammation. ${ }^{8}$ Thirty patients received radiation and 29 patients were treated with sham irradiation. There was no significant difference in soft tissue inflammation between the two groups at 24 weeks. This study did not treat patients with oral prednisone as we do and did not provide sufficient follow up information. External beam irradiation initially causes exacerbation of inflammation in TO. Since concomitant prednisone was not utilised, side effects of the radiation such as short term increased inflammation, lid swelling, chemosis, and proptosis would obscure any improvement in soft tissue inflammation. A prospective study comparing placebo and sham irradiation with prednisone and external beam radiation, evaluated for a minimum of 6 months, is needed.

\section{Optic neuropathy}

The optic nerve has a serpiginous course that allows for several millimetres of proptosis before compromise of optic nerve function. Proptosis, then, serves a protective function by expanding the total orbital volume. The orbital apex, conversely, has no room for expansion. Muscle enlargement posteriorly results in compression of the optic nerve just anterior to the optic canal. Muscle enlargement in the absence of significant proptosis is most likely to promote optic nerve compression. It is essential to check for signs of afferent dysfunction (for example, decreased visual acuity, abnormal colour vision, afferent pupillary defect, and abnormal visual fields) even in apparently asymptomatic patients. The process is often bilateral, so visual field testing remains essential even in the absence of an afferent pupillary defect. The most common clinical presentation is gradual onset of central visual loss, a central scotoma, and a normal optic nerve head appearance in a patient with mild to moderate proptosis.

If the clinical examination reveals afferent dysfunction, an orbital CT scan (without contrast) should be obtained in a timely fashion. Both axial and direct coronal views should be obtained to clarify the optic nerve position with respect to the extraocular muscles. Crowding of the optic nerve at the orbital apex is diagnostic.

If optic nerve compression is identified, corticosteroids are used in a temporising fashion until radiation or surgical decompression is performed. High dose steroids (at least $80 \mathrm{mg}$ prednisone) should be initiated immediately. In our experience, if the orbit is acutely inflamed, steroids will result in marked visual improvement within 48 hours. Unfortunately steroids are usually only temporarily effective in treating optic neuropathy and significant side effects preclude long term use.

External beam radiation (20 Gy) is the definitive intervention in the actively inflamed orbit with visual loss as a result of optic nerve compression. If the visual loss has been precipitous the patient should be treated with intravenous corticosteroids and radiation initiated immediately to prevent permanent visual dysfunction. External beam is also effective in patients who have undergone orbital decompression but have not achieved complete resolution of the optic nerve impairment. ${ }^{312-16}$

Radiation is contraindicated in diabetics and children owing to the increased risk of radiation retinopathy. External beam radiation causes temporary ocular irritation and exacerbates soft tissue inflammation from approximately 2 weeks following therapy and continuing for at least 24-36 weeks. Oral corticosteroids should, therefore, be continued during the course of radiation

Debate 


\section{Debate}

and tapered slowly over 3 months. If vision deteriorates despite corticosteroids and radiation treatment, surgical decompression is warranted. Conversely, if visual status is only temporarily improved with surgical decompression, external beam radiation should be performed.

\section{Conclusions}

External beam radiation is a safe and effective intervention in non-diabetic adults with acute congestive dysthyroid orbitopathy. Appropriate patient selection is essential. Orbital inflammatory signs and symptoms are eliminated within weeks of the therapy when radiation is combined with oral corticosteroids. Optic nerve compression is alleviated within 1 month. Only corticosteroids, with their significant side effects, provide a similar direct intervention in the acute disease process.

Clinical studies have consistently demonstrated that radiation, especially in combination with oral prednisone, is effective at decreasing soft tissue signs and symptoms due to inflammation. Radiation is also widely used for treatment of visual loss in patients with actively inflamed orbits. Proptosis and dysmotility are inconsistently improved with radiotherapy; external beam radiation should not be used for these indications where chronic fibrosis is probable.

A large multicentre trial is needed to confirm the clinical impression that radiation can minimise the active inflammatory phase in moderate to severely affected patients with TO. We are currently evaluating the long term outcome of thyroid associated optic nerve compression treated with radiation versus orbital decompression.

\section{REFERENCES}

1 Barsouk A, Peele KA, Kilianski J, et al.

Antibody-dependent cell-mediated cytotoxicity against

orbital target cells in thyroid-associated ophthalmopathy and related disorders. J Endocrin Invest

1996;19:334-41.

2 Wall JR, Barsouk A, Stolarski C, et al. Serum muscle antibodies predicted the development of ophthalmopathy in a euthyroid subject with a family history of autoimmunity. Thyroid 1996;6:353-8.

3 Peele-Cockerham KA, Kennerdell JS.

Thyroid-associated orbitopathy. Focal Points 1997;XV(1): $1-14$

4 Bartalena L, Marcocci C, Chiovato L, et al. Orbital cobalt irradiation combined with systemic corticosteroids for Graves' ophthalmopathy: comparison with systemic corticosteroids alone. J Clin Endocrinol Metab 1983;56: $1139-44$

5 Hurbli T, Char DH, Harris J, et al. Radiation therapy for thyroid eye diseases. Am J Ophthalmol 1985;99:633-7.

6 Peterson IA, Kriss JP, McDougall IR, et al. Prognostic factors in the radiotherapy of Graves' ophthalmopathy Int I Radiat Oncol Biol Phys 1990;19:159-64.

7 Prummel MF, Mourtis MP, Blank L, et al. Randomized double-blind trial of prednisone versus radiotherapy in Graves' ophthalmopathy. Lancet 1993;342:949-5.

8 Mourits MPh, Van Kempen-Harteveld ML, Garcia Garcia MB, et al. Radiotherapy for Graves' orbitopathy: randomized placebo-controlled study. Lancet 2000;355:1501-9.

9 Kennerdell JS, Maroon JC, Buerger GF. Comprehensive surgical management of proptosis in dysthyroid orbitopathy. Orbit 1987;6:153-79.

10 Sakata K, Hareyama M, Oouchi A, et al. Radiotherapy in the management of Graves' ophthalmopathy. Jap J Clin Oncol 1998;28:364-7.

11 Donaldson SS, Bagshaw MA, Kriss JP. Supervoltage radiotherapy for Graves' ophthalmopathy. J Clin Endocrinol Metab 1973;37:276-85

12 Tagami T, Tanaka K, Nakamura H, et al. High-dose intravenous steroid pulse therapy in thyroid-associated ophthalmopathy. Endocrine J 1996;43:689-99.

13 Matejka G, Verges B, Vaillant G, et al. Intravenous methylprednisolone pulse therapy in the treatment of Graves' ophthalmopathy. Hormone Metab Res 1998;30:93-8.

14 Beckendorf V, Maalouf T, George J-L, et al. Place of radiotherapy in the treatment of Graves'orbitopathy. Int J Radiation Oncology Biol Phys 1999:43:805-15.

15 Wilson WB, Prochoda M. Radiotherapy for thyroid orbitopathy. Arch Ophthalmol 1995;113:1420-5.

16 Kao SCS, Kendler DL, Nugent RA, et al. Radiotherapy in the management of thyroid orbitopathy. Arch Ophthalmol $1993 ; 111: 819-23$

\title{
Does radiotherapy have a role in the management of thyroid orbitopathy? View 2
}

\author{
Maarten Ph Mourits
}

\section{Ph Mourits, Department of Ophthalmology, University Hospital Utrecht, Heidelberglaan 100, PO Box 85500, 3508 GA Utrecht, Netherlands}

T hyroid orbitopathy (TO) is an autoimmune disorder characterised by eyelid swelling and retraction, proptosis, restrictive motility disturbances and, in severe cases, compression of the optic nerve. TO can precede, coincide, or follow Graves' thyroid disease. The exact cause of TO remains unclear, but the pathophysiological mechanism is understood. ${ }^{1}$ Activated lymphocytes infiltrate the orbital tissues and stimulate fibroblasts within the orbit to generate highly hydrophilic glycosaminoglycans, which cause swelling of the extraocular muscles and peribulbar connective tissues (for example, fat). The bony confines of the orbit allow minimal expansion of the orbital contents, thus they are pushed forward as much as the rigidity of the orbital septum permits. ${ }^{2}$ The optic nerve then becomes compressed either directly by enlarged muscles or as a consequence of raised intraorbital pressure. Swelling and stretching of the extraocular muscles cause inability to relax and subsequent restriction of gaze in the contralateral direction of the enlarged muscle. Similarly, involvement of the levator palpebrae and of the lower lid retractors result in eyelid retraction. Secondary changes as a result of activation of orbital fibroblasts are formation of fibrotic tissues within the fibromuscular framework of the orbit causing these alterations to become irreversible. Primary and secondary changes can be found in the same tissue biopsy. 
The rationale for radiotherapy in thyroid eye disease is the high sensitivity of lymphocytes and fibroblasts to ionising radiation. ${ }^{3}$ Radiotherapy was first used in the 1940s to irradiate the pituitary gland in patients with thyroid eye disease, as it was thought that pituitary hormonal factors caused exophthalmos. ${ }^{45}$ Only patients who had received inadvertent orbital irradiation, as a consequence of badly collimated beams, improved. ${ }^{67}$ Thus treatment evolved to selective irradiation of the retrobulbar structures.

The results of a recent questionnaire, sent to 1348 institutes, reveal that radiotherapy remains a well accepted and frequently used treatment for TO. ${ }^{8}$ Radiotherapy for TO is applied using a megavoltage linear accelerator that delivers a high energy, low scattered, well collimated beam, and the treatment is given as $8-10$ daily fractions of 2 Gy over a period of 2 weeks.' Radiotherapy is contraindicated in patients with diabetes mellitus because of the risk of induction or aggravation of retinopathy. ${ }^{10}$ The major advantage of the radiation treatment is the lack of complications. However, the question remains "Is it really effective?"

There are several factors hampering proper evaluation of treatment results in patients with TO:

- TO is a self limiting disease that passes through several phases: progressive deterioration (active phase of the disease), plateau, partial improvement, burnt out phase. ${ }^{11}$ The length of these phases can be extremely variable

- The expression of TO is also extremely variable; many features are not consistently present

- Objective assessment criteria are lacking or vary $^{12}$ (for example, type of exophthalmometer used)

- Factors such as thyroid status, ${ }^{13}$ smoking habits, ${ }^{14}$ concomitant diabetes mellitus, ${ }^{15}$ and age may influence the natural history and become confounding variables in comparative studies.

It is within this framework that we have to judge studies on the efficacy of retrobulbar irradiation.

Donaldson $e t a l^{16}$ in 1973, were the first to describe the results of 10 fractions of 200 rads delivered by a $4.8 \mathrm{meV}$ linear accelerator. Of 23 patients with TO, seven had a marked improvement in major symptoms and signs and eight a good response, while worsening of the ophthalmopathy did not occur. Decrease in eyelid and conjunctiva swelling was noted together with decrease in proptosis. Improvement in ocular movement was rare. The authors considered severe visual acuity loss not a contraindication for radiotherapy. The best results were found in patients, in whom irradiation was given shortly after the onset of eye symptoms. Additionally, patients who had not responded to oral steroids improved after radiotherapy. Bartalena et $a l^{17}$ compared orbital cobalt irradiation plus oral steroids with oral steroids alone and found a better response in patients who had received the combined treatment. Similar results have been described by many others, ${ }^{18}{ }^{19}$ all of them stressing the point that patients in the active phase of their TO respond better than patients with longstanding and burnt out orbitopathy. In general, these studies showed an improvement in two thirds of the patients. The best response was seen in eyelid swelling, modest improvement occurred in ocular motility and visual acuity, and there was little effect on proptosis. ${ }^{20}$ These studies were retrospective and not randomised. Some patients had abnormal thyroid function, many had been treated or were being treated with corticosteroids at the time of the study, the interval between start of treatment and assessment of treatment outcome was variable, and the methods of assessment were variable and poorly described. These factors may explain the differences in outcomes. One aspect is consistent in all these studies-a considerable number of patients needed additional surgery after "successful" radiotherapy.

Prummel et $\mathrm{l}^{21}$ were the first to deliver evidence based data for the efficacy of radiotherapy in TO by performing a prospective, randomised study in which 28 patients received radiotherapy plus a placebo and 28 sham irradiation plus oral prednisone. Strict inclusion and exclusion criteria were used, a fixed interval between start of treatment and of assessment of treatment result was defined, and the methods of clinical assessment and outcome were standardised. The responses to prednisone $(14 / 28 ; 50 \%)$ and to radiotherapy (13/ $28 ; 47 \%$ ) were similar. Improvements were seen in soft tissue involvement and in ocular motility, but not in proptosis. Changes in visual acuity did not occur; however, patients with reduced vision were excluded from the study. Prummel et al concluded that radiotherapy was the treatment of choice for patients with moderately severe TO because of the absence of complications after radiotherapy and frequently occurring side effects of prednisone. One of the drawbacks of this study was that it compared the efficacy of radiotherapy with prednisone, which has never been tested for efficacy against a placebo. There is circumstantial evidence of the efficacy of corticosteroids from a prospective, randomised, masked clinical trial that compared treatment with prednisone to treatment with cyclosporine in patients with severe TO. ${ }^{22}$ After a period of 12 weeks, $61 \%$ of the prednisone treated versus $22 \%$ of the cyclosporine treated patients responded to therapy $(\mathrm{p}=$ 0.018 ). Assuming that cyclosporine has no detrimental effect, this study shows that prednisone is efficacious. When radiotherapy is as effective as prednisone, radiation can then be regarded as efficacious.

In a rigorously controlled randomised clinical trial using patients with moderately severe orbitopathy, we compared radiotherapy with sham irradiation and could not demonstrate improvement in soft tissue signs, proptosis, or ocular motility. ${ }^{23-25}$ Seventy five per cent of the irradiated patients required additional strabismus surgery. The study design excluded patients with severe eye disease such as patients with optic nerve compression, and therefore the efficacy of radiotherapy remains unclear in this subgroup. The potential ability of radiotherapy (or of corticosteroids) is to reduce the period of disease activity in TO and to allow rehabilitative surgery more quickly after the onset of the disease. As reliable parameters for the assessment of disease activity are controversial such a study is difficult to undertake at present. Our study, using the clinical activity score as index of disease activity, ${ }^{26}$ showed

Debate 


\section{Debate}

a more rapid fall of disease activity after radiotherapy than after sham irradiation.

In conclusion, with the introduction of more advanced, evidence based clinical trials, the initial enthusiasm about the efficacy of radiation for TO has been replaced by scepticism. At present, orbital radiotherapy remains useful in the treatment of a select number of patients with $\mathrm{TO}$, but overall its place in management is uncertain

\section{REFERENCES}

1 Burch HB, Wartofsky L. Graves' ophthalmopathy: current concepts regarding pathogenesis and management. Endocrinol Rev 1993;14:747-93.

2 Koornneef L. Eyelid and orbital fascial attachments and their clinical significance. Eye 1988;2:130-4.

3 Sautter-Bihl ML. Orbital radiotherapy: recent experience in Europe. In: Wall JR, How J, eds. Graves' ophthalmopathy. Boston: Blackwell, 1990: 145-57.

4 Ginsburg S. Postoperative progressive exophthalmos, with low basal metabolic rate. Ann Intern Med $1939 \cdot 13: 424-50$.

5 Mandeville FB. Röntgen therapy of orbital-pituitary portals for progressive exophthalmos following subtotal thyroidectomy. Radiology 1943;41:268-71.

6 Jones A. Orbital X-ray therapy of progressive exophthalmos. Br J Radiol 1951;24:637-46.

7 Beierwaltes WH. X-ray treatment of malignant exophthalmos: a report of 28 patients. J Clin Endocrinol 1953;13:1090-100

8 Leer JWH, van Houte P, Davelaar J. Indications and treatment schedules for irradiation of benign diseases: a survey. Radiather Oncol 1998;48:249-57.

9 Kahaly GJ, Gorman CA, Kal HB, et al. Radiotherapy for Graves' ophthalmopathy. In: Prummel MF, Wiersinga WM, Mourits MPh, Heufelder AE, eds. Recent developments in Graves' ophthalmopathy. London: Kluwer Academic Publishers 2000: 1 15-31

10 Viebahn M, Marricks ME, Osterloh MD. Synergism between diabetic and radiation retinopathy: case report and review. Br J Ophthalmol 1991;75:29-32.

11 Rundle FF, Wilson CW. Development and course of exophthalmos and ophthalmoplegia in Graves' disease with special reference to the effect of thyroidectomy. Clin Sci 1945; 5: 177-94

12 Gorman CA. The measurement of change in Graves ophthalmopathy. Thyroid 1998;8:539-43.
13 Prummel MF, Wiersinga WM, Mourits MPh, et al. Amelioration of eye changes of Graves' ophthalmopathy by achieving euthyroidism. Acta Endocrinol 1989;121:185-89.

14 Prummel MF, Wiersinga WM. Smoking and risk of Graves' disease. JAMA 1993;269:479-82.

15 Kalmann R, Mourits MPh. Diabetes mellitus: a risk factor in patients with Graves' orbitopathy. Br J Ophthalmol 1999;83:463-5.

16 Donaldson SS, Bagshaw MA, Kriss JP. Supervoltage radiotherapy for Graves' ophthalmopathy. J Clin Endocrinol Metab 1973;37:276-85.

17 Bartalena L, Marcocci C, Chiovato L, et al. Orbital cobalt irradiation combined with systemic corticosteroids for Graves' ophthalmopathy: comparison with systemic corticosteroids alone. J Clin Endocrinol Metab 1983;56: $1139-44$

18 Hurbli T, Char DH, Harris J, et al. Radiation therapy for thyroid eye diseases. Am J Ophthalmol 1985;99:633-7.

19 Petersen IA, Kriss JP, McDougall IR, et al. Prognostic factors in the radiotherapy of Graves' ophthalmopathy Int J Radiat Oncol Biol Phys 1990;19:159-64.

20 Bahn RS, Gorman CA. Choice of therapy and criteria for assessing treatment outcome in thyroid associated ophthalmopathy. Endocrinol Metab Clin N Am 1987; 16:391-407.

21 Prummel MF, Mourits MPh, Blank L, et al. Randomised double-blind trial of prednisone versus radiotherapy in Graves' ophthalmopathy. Lancet 1993;342:949-54.

22 Prummel MF, Mourits MPh, Berghout A, et al. Prednisone and cyclosporine in the treatment of severe Graves' ophthalmopathy. N Engl J Med 1989;321:1353-9.

23 Mourits MPh, Van Kempen-Harteveld ML, García García $M B$, et al. Radiotherapy for Graves'orbitopathy: randomised placebo-controlled study. Lancet 2000;355: 1505-9.

24 Pinchera A, Wiersinga WM. Recommendations for the assessment of therapeutic outcome of Graves' disease in clinical trials. In: Prummel MF, Wiersinga WM, Mourits $\mathrm{MPh}$, Heufelder AE, eds. Recent developments in Graves ophthalmopathy. London: Kluwer Academic Publishers, 2000:81-7.

25 Gerding MN, Prummel MF, Kalman R, et al. The use of colour slides in the assessment of changes in soft-tissue involvement in Graves' ophthalmopathy. J Endocrinolol Invest 1998;21:459-62

26 Mourits MPh, Koornnef L, Wiersinga WM, et al. Clinical criteria for the assessment of disease activity in Graves' ophthalmopathy: a novel approach. Br J Ophthalmol 1989;73:639-44.

\title{
Does radiotherapy have a role in the management of thyroid orbitopathy? Comment
}

\author{
Alan A McNab
}

\section{A A McNab,}

Lead clinician Orbital, Plastic and Lacrimal Clinic, Roya Victorian Eye and Ear Hospital, Melbourne, Australia
D espite extensive research, thyroid orbitopathy remains a problematic disease. Until recently, there has been little understanding of the pathophysiology of such a common, long recognised autoimmune disease. Thyroid orbitopathy has a broad range of clinical manifestations and a highly variable natural history. Figure l shows a patient with acute onset, severe thyroid orbitopathy who had significant corneal exposure and required urgent levator recessions to protect the corneas. She was treated with oral prednisolone followed by orbital irradiation. Over a period of some months, the signs of acute inflammation settled, and eyelid and conjunctival swelling diminished (Fig 2). Was this due to the irradiation, the prednisolone, a combination of

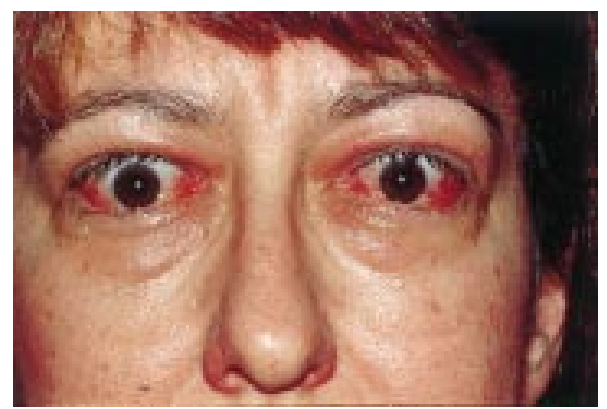

Figure 1 A 40 year old woman with acute onset moderately severe thyroid orbitopathy, whose main problem was corneal exposure. 


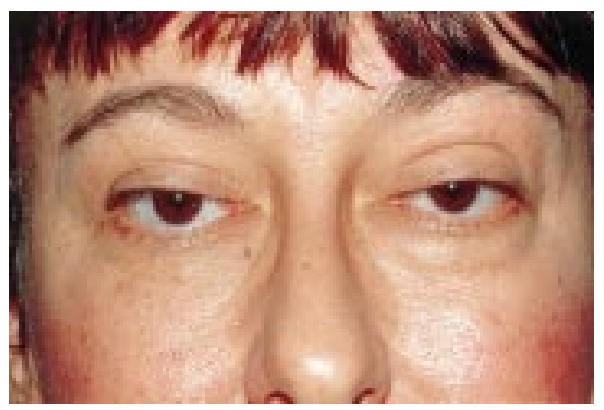

Figure 2 The same patient several months after urgent levator recessions, oral prednisolone and orbital irradiation. Is the improvement due to the steroids, the irradiation, both, or just the natural history of the disease?

the two, or merely the natural history of the disease? We do not know.

There are no really effective treatments and current treatments rely on systemic immunosuppression, orbital irradiation, and surgery to relieve the mechanical effects of the disease such as optic nerve compression, proptosis, strabismus, and lid retraction and swelling. Future therapies aimed at the molecular and immunological basis of the disease are more likely to be of benefit. A key component of such therapy will be the ability to detect the disease at its earliest stage or detect a predisposition to develop thyroid orbitopathy. Until that day, we are reliant on immunosuppression, irradiation, and surgery.

Orbital irradiation for thyroid orbitopathy has a relatively long history. Like many therapies in medicine, its use has been based on slender evidence. There are divergent views on its efficacy, but as indicated in Maarten Mourits's review, it remains widely used. This probably says more about the lack of any effective alternative treatments than the effectiveness of orbital irradiation

If evidence based criteria are used, the evidence for a positive effect from orbital irradiation in thyroid orbitopathy is very limited. There are no good studies of its effectiveness in those patients with the most severe forms of thyroid orbitopathy with optic neuropathy. Radiotherapy has little if any effect on proptosis. The evidence of its efficacy in soft tissue signs is conflicting. There is a marginal benefit in ocular motility. Despite this, radiotherapy has its strong advocates and many of us who remain sceptical about its effectiveness still use it at times because of the lack of other safe therapies in patients whose eyes and lives are ruined by this disease.

As the guest experts point out, there is room for more studies on the role of radiotherapy, alone or in combination with other treatments. As radiotherapy seems to be most effective early in the course of thyroid eye disease, it would be helpful if the onset of disease could be detected earlier, and predictors of its severity and time course could be identified. This might allow for earlier treatment, which may also prove more effective. It is to be hoped, however, that novel forms of therapy aimed at the molecular basis of the disease will overtake such efforts and make orbital irradiation and this type of discussion redundant.

\section{ADDENDUM}

Since the manuscripts for this series were prepared, an important study examining the effectiveness of radiotherapy in thyroid orbitopathy has been published. ${ }^{1}$ Gorman et al reported on the results of a prospective, randomised, internally controlled, double blind clinical trial evaluating the effectiveness of 20 Gy external beam irradiation to one orbit. They reported no clinically or statistically significant difference between the treated and untreated orbit at 6 months, and a marginal reduction in proptosis and muscle volume at 12 months, which may have been the result of natural remission or radiotherapy. Although this study has been criticised ${ }^{2}$ for including many patients whose disease had been present for over 6 months, it does provide further evidence of the limited value of radiotherapy for this disease. As stated above, a study looking at radiation given in the early months of the disease would be helpful in establishing which patients, if any, would benefit.

\section{REFERENCES}

1 Gorman CA, Garrity JA, Fatourechi V, et al. A prospective, randomised, double-blind, placebo-controlled study of orbital radiotherapy for Graves' ophthalmopathy. Ophthalmology 2001;108:1523-34.

2 Feldon SE. Radiation therapy for Graves' ophthalmopathy: trick or treat? [Editorial] Ophthalmology 2001;108:1521-2. 